\title{
Management of cystic pancreatic lesions
}

\author{
Klaus Sahora $\cdot$ Martin Schindl
}

Received: 28 January 2019 / Accepted: 12 March 2019 / Published online: 8 May 2019

(C) The Author(s) 2019

\begin{abstract}
Summary
Background Three decades ago, cystic neoplasia of the pancreas were a relatively unknown and poorly classified tumor entity. Nowadays, they account for a large proportion of treated lesions in the field of pancreatic surgery. Depending on the cyst type and morphology, a conservative and observational treatment approach has become standard in selected cases. Cystic tumors of the pancreas include both, neoplastic cysts with a variable malignant potential and pseudocysts, which occur as a consequence of a previous pancreatis.

Methods Contrast-enhanced multiphase computed tomographic (CT) scan, magnetic resonance imaging (MRI) with the possibility of non-invasive cholangiopancreatography (MRCP) and endoscopic ultrasound (EUS) are the key methods for detailed diagnostic evaluation and risk assessment of cystic pancreatic tumors.

Results Treatment of cystic tumors of the pancreas is safe according the published guidelines.

Conclusion In patients with a confirmed diagnosis of the cyst subtype, pancreatic resection should be performed in surgically fit patients for intraductal papillary mucinous neoplasia (IPMN) with main duct and mixed type appearance, mucinous cystic neoplasia (MCN) and solid pseudopapillary neoplasia (SPN) regardless their size.
\end{abstract}

Assoc. Prof. PD Dr. K. Sahora ( $\varangle)$ · Univ. Prof. Dr. M. Schindl

Universitätsklinik für Chirurgie, Comprehensive Cancer

Center Vienna, Medizinische Universität Wien,

Währinger Gürtel 18-20, 1090 Vienna, Austria

Klaus.sahora@meduniwien.ac.at
Keywords Cyst · Pancreas · Guidelines · Surgery · Observation

\section{Main novel aspects}

- This review is based on the latest guidelines and publications in the field of pancreatology.

\section{Introduction}

Three decades ago, cystic neoplasia of the pancreas were a relatively unknown and poorly classified tumor entity. Nowadays, they account for a large proportion of treated lesions in the field of pancreatic surgery. The development of high-resolution sectional imaging methods and their widespread availability has led to a considerable increase in their diagnosis [1]. While most of these lesions were surgically removed in the past, increasing knowledge of the biological behavior of these cysts has changed management in many patients [2]. Depending on cyst type and morphology, a conservative and observational treatment approach has become standard in selected cases $[3,4]$. This shift of paradigm was based on the observations that (1) cystic lesions, such as serous cystic neoplasia (SCN), do not progress to invasive cancer, (2) subtypes of intraductal papillary mucinous neoplasia (IPMN) show a slow adenoma-carcinoma transformation that takes many years to occur, and (3) premalignant and malignant cystic lesions of the pancreas usually have radiomorphologic features that distinguish them from benign diseases [5]. Overall, one can assume that the risk that a cystic lesion is malignant at the time of diagnosis does not exceed $0.21 \%$ for cystic neoplasms $>2 \mathrm{~cm}$ [6]. Therefore, the decision for resection or observation is based on the diagnosis of the subgroup of the cystic lesion, the early detection of suspicious morphologic changes and cyst-associated symptoms, 
as well as patient age and comorbidities. If it is not possible to initially determine the subtype, frequently because of small cyst size, the further therapeutic approach is based on radiomorphologic criteria and the patient's symptoms.

\section{Non-neoplastic cystic lesions}

Cystic tumors of the pancreas include both neoplastic cysts with a variable malignant potential and pseudocysts, which occur as a consequence of a previous pancreatitis. The cyst content is necrotic debris to clear serous fluid (amylase and lipase rich as a result of main- or branch-duct communication) and the cyst wall is usually thin without epithelial lining, which is a fundamental feature to distinguish them from neoplastic cysts.

\section{Neoplastic cystic lesions}

\section{Serous cystic neoplasia}

SCN represent approximately $30 \%$ of neoplastic cystic tumors. Women are more frequently affected than men (65 vs. 35\%), with an average age of 62 years. Most of these lesions (more than 50\%) are localized in the head of the pancreas. SCN are predominantly lined by a single-row isoprismatic epithelium and contain a clear serous fluid. Depending on the size and number of cysts, a distinction is made between serous microcystic adenomas and rare serous oligocystic adenomas. Most SCN occur as microcystic adenomas built of numerous cysts only a few millimeters in size, which give the tumor a sponge-like appearance. Often a star-shaped scar is recognizable in the center. SCN are benign neoplasms that have no risk to malignant progression [2].

\section{Mucinous cystic neoplasia}

Mucinous cystic neoplasia (MCN) are usually found by chance in middle-aged women, representing 44 to $49 \%$ of all cystic pancreatic tumors. MCN in men have not been reported. According to the new WHO classification, MCNs are defined as followed: cystic tumors without connection to the pancreatic duct system, which have mucus-producing epithelium and an ovarian-like stroma. The typical localizations are body and tail of the pancreas. The average size at diagnosis is $>5 \mathrm{~cm}$. In about $10 \%$ of cases, an invasive carcinoma is found at diagnosis. MCN are characterized by cysts lined with highly prismatic, mucus-producing cells. The cysts themselves are filled with mucus. The distinctive feature compared to intraductal papillary mucinous neoplasms is that the latter have a connection to the pancreatic duct system. MCN present as unilocular or multilocular cysts. Due to the uncertain dignity of MCN with a certain malignant potential, surgical resection is recommended $[7,8]$. The prog- nosis after complete resection of noninvasive MCN is excellent. In patients with invasive MCN carcinoma, the 5 -year survival rate is between 30 and $63 \%$, with depth of invasion being the most important prognostic factor [7].

\section{Intraductal papillary mucinous neoplasia}

IPMN represent 20 to $25 \%$ of pancreatic cysts, with an increasing incidence $[9,10]$. The average age of patients is 65 years and older. There is no clear gender dominance but there are geographic variations. IPMNs are characterized by papillary proliferation of mucus-producing cells within one or more communicating ducts. Clinically, IPMN may present as recurrent pancreatitis due to the production of a tough mucus that clogs the main pancreatic duct. The distinction between main- and branch-duct IPMN is important in determining conservative or surgical treatment. While main-duct IPMN converts into carcinoma in 60 to $92 \%$ of cases, the likelihood of a malignant side-branch IPMN is significantly lower (6 to $46 \%$ ) [11, 12]. Immunohistochemically, IPMN can be further subclassified into intestinal, pancreaticobiliary, gastric, and oncocytic subtypes and tubular, colloid, or oncocytic patterns for malignant IPMNs. The prognosis of IPMN carcinoma varies between the different types. Carcinoma of the tubular subtype has a poor prognosis similar to ductal adenocarcinoma, whereas carcinomas of the colloid and oncocytic subtypes show a significantly better 5-year survival of 65 to $80 \%$ [13].

\section{Solid pseudopapillary neoplasia}

Solid pseudopapillary neoplasia (SPN) or "Frantz tumor" are mainly diagnosed in women in their forties but can be diagnosed at any age. There is no predisposition for their localization to a specific part of the pancreas. SPN are composed of solid tumor and necrotic cystic areas. These contain detritus and blood and therefore differ from the construction of a true cystic neoplasm, as they lack a proper epithelial lining. SPN have an inherent risk of malignancy, with vascular and perineural invasion and metastatic spread in about $10-15 \%$ at initial diagnosis. Thus, there is a clear indication for surgery. After complete removal of the primary tumor and (if present) metastases, the prognosis is excellent.

Beside these major representatives of pancreatic cystic neoplasms, all entities of pancreatic tumors, including ductal adenocarcinoma, may present with a cystic degenerative necrotic appearance. Moreover, less frequently diagnosed congenital cysts, lymphoepithelial cysts, and squamoid cystic changes of the pancreatic duct show no malignant potential. 


\section{Diagnosis}

Contrast-enhanced multiphase computed tomographic (CT) scan, magnetic resonance imaging (MRI) with the possibility of noninvasive cholangiopancreatography (MRCP), and endoscopic ultrasound (EUS) are the key methods for detailed diagnostic evaluation and risk assessment of cystic pancreatic tumors. MRI has the advantage of differentiating small lesions and detecting pancreatic duct communication and septa as well as the solid part. The differentiation of IPMN and MCN by MRI using diffusion-weighted sequences and secretin-enhanced MRCP is the method of choice. In addition, EUS can provide information on the morphology of small cystic lesions and offers the possibility of fine-needle aspiration. Further cytological/laboratory analysis (carcinoembryonic antigen, CEA, and mucin) of the cystic aspirate should be performed. CEA and the detection of mucin provide valuable information regarding the differentiation of mucinous (IPMN, MCN) from non-mucinous lesions (SCN, SPN, pseudocyst) [14]. Endoscopic retrograde cholangiopancreatography (ERCP) has been largely replaced by noninvasive MRCP, but still has some value in the diagnosis of a main-duct IPMN vs. chronic pancreatitis where it is used with microprobe "spy-glass" videoendoscopy for direct visualization of the duct. New fused radiological methods such as F-18 fluorodeoxyglucose positron-emission tomography with CT or MR are of minor importance in the primary diagnosis of cystic lesions of the pancreas.

The inclusion of gender, patient age, radiomorphologic changes, and analysis of cystic aspirate permits an accurate classification of cystic lesions in the majority of patients. CEA values $>192 \mathrm{ng} / \mathrm{ml}$ and/or viscous (spinning test-positive) cyst content are pathognomonic for IPMN and MCN. If the patient is male, the diagnosis is likely IPMN. If there is a radiologically confirmed connection to the pancreatic duct system and/or if the cyst fluid is positive for amylase, the diagnosis of an IPMN can also be made in female patients. Pseudocysts also contain amylase-rich fluid, but this is negative in the spinning test (no mucin) and contains a low concentration of CEA. However, IPMN with low CEA concentration and non-mucinous cyst content do exist in up to $20 \%$ [15]. Patient history is of particular importance. If no episode of pancreatitis or upper abdominal trauma is reported, a pseudocyst is relatively unlikely. SCN are distinguished by the lack of amylase in the cyst aspirate and a lack of duct communication. Spongy microcystic appearance and a typical central star-shaped scar, often calcified, are almost exclusively observed in SCN. However, rare oligocystic SCNs, which can easily be held to be MCN or side-branch IPMNs, are known "pitfalls" during differential diagnosis. Also, the very compact microcystic morphology of SCN can complicate the differential diagnosis of SPN, especially in small lesions $<3 \mathrm{~cm}$, because of its radiologically similar appearance.
Regardless of the cyst entity, invasive cystic carcinoma is often associated with the following "highrisk stigmata": duct dilation $>10 \mathrm{~mm}$ and/or duct irregularity, a contrast-enhancing solid mass or nodule, jaundice, peripheral calcification. Further socalled "worrisome features" are suspicious of an early stage of malignant transformation: cyst size $>3 \mathrm{~cm}$, an abrupt change of duct diameter, a main duct diameter of $5-9 \mathrm{~mm}$, solid cystic components $<5 \mathrm{~mm}$, thickened cyst wall, size progression of $>5 \mathrm{~mm}$ over 2 years, local lymphadenopathy and elevated serum CA 19-9 level.

\section{Observation vs. resection}

In patients with a confirmed diagnosis of the cyst subtype, pancreatic resection should be performed in surgically fit patients for IPMN with main-duct and mixed-type appearance, for MCN and SPN regardless of their size [4, 16-18]. Surgery for SCN should be limited to patients with symptoms because of cystic growth such as inappetence, bloating, or unspecific upper abdominal pain.

A conservative approach is justified in asymptomatic patients with branch-duct IPMN without suspicious radiomorphologic or cytological changes, as well as in patients with small $(<3 \mathrm{~cm})$ non-classifiable cystic lesions in the absence of symptoms and worrisome features. The guidelines of the International Association of Pancreatology 2017 provide a detailed guide to managing these patients [17]. As recent studies have shown, observance in surgically fit patients should be life-long, as malignant progression was still observed in IPMN which had not shown any growth or suspicious morphologic changes for 5-10 years. Overall, it can be assumed that the cumulative risk of pancreatic carcinoma in patients with IPMN is 18 times higher than in the normal population [19].

\section{Operative strategy}

The basic surgical techniques for cystic tumors of the pancreas have not changed significantly in the past decades. Since IPMN frequently spread across the entire pancreas, the extent of pancreatic resection and the need for a total pancreatectomy must be discussed individually. If there are no signs of malignancy in preoperative imaging and the clinical presentation of the patient, a parenchyma-sparing resection, such as middle pancreatectomy, enucleation, or distal pancreatectomy without splenectomy can be considered. A major challenge for both surgeons and pathologists are main-duct IPMN. The presence of "skip lesions," i.e., duct segments with normal epithelial lining close to pathologically altered epithelial segments, must be considered when determining the extent of resection. It is helpful to send a piece of 3 to $4 \mathrm{~mm}$ of the pancreatic resection margin as a frozen section early during surgery, which gives the pathologist ample time for ex- 


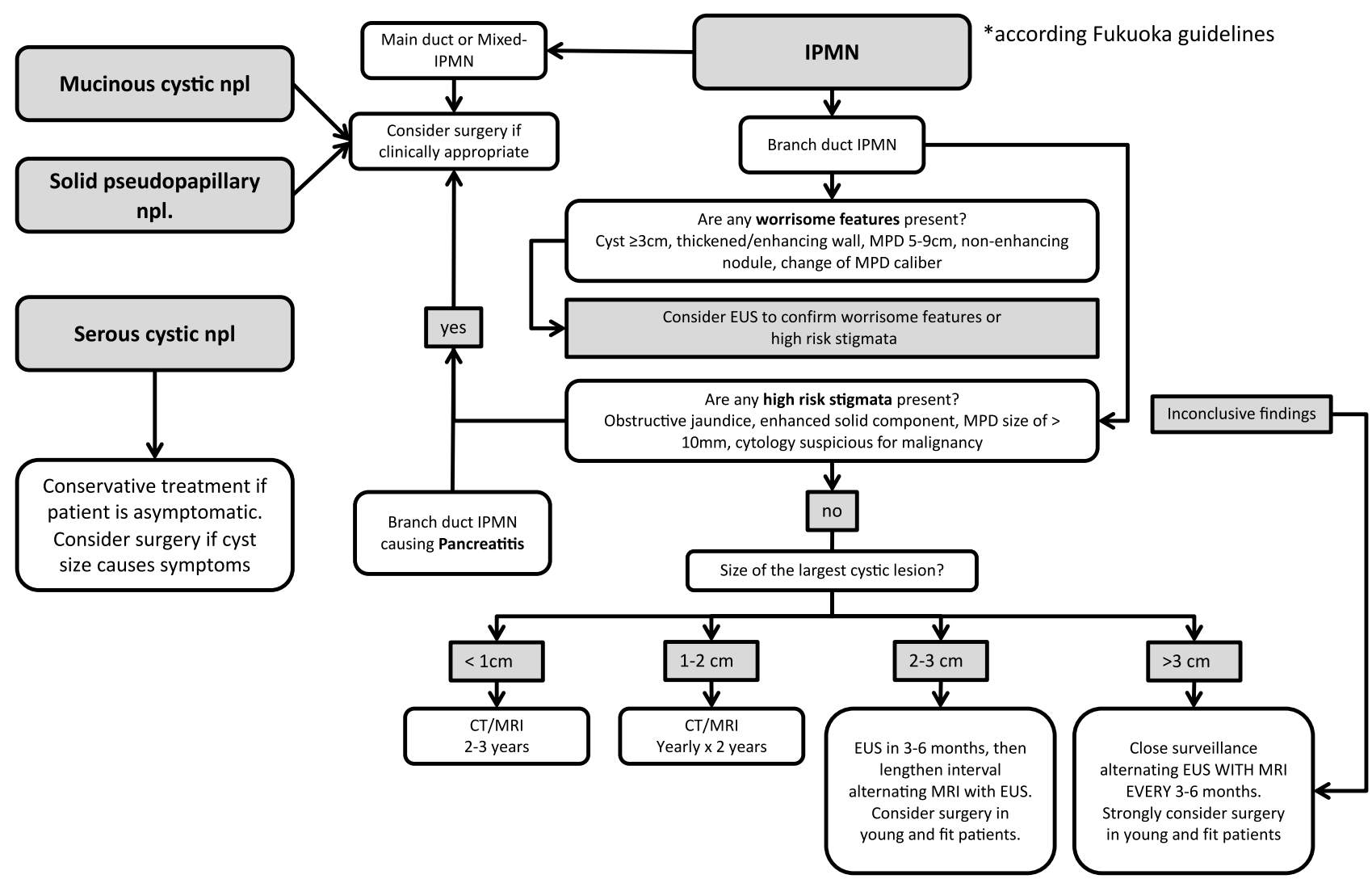

Fig. 1 Recommended approach in patients with cystic neoplasms. EUS endoscopic ultrasound, MRI magnet-resonance imaging, CT computed tomography, IPMN intraductal papillary mucinous neoplasm, NPL neoplasm

amination. If the resection margin shows high-grade dysplasia of the epithelium, the resection should be extended until a clear resection margin is confirmed. Intraoperative endoscopy of the dilated main pancreatic duct can be useful for identifying skip lesions and determining the resection margin.

\section{Follow-up}

In patients with benign IPMN and negative resection margins, a follow-up at 2 and 5 years is generally recommended. If the histological work-up reveals moderate or severe dysplasia at the resection margin, frequent radiologic controls should be performed, primarily every 6 months by MRI, CT, or EUS [17]. In invasive IPMN carcinoma, the follow-up regimen is identical to that of pancreatic ductal adenocarcinoma. When single cysts of multifocal branch IPMN were left in the pancreatic remnant, further observance follows the same regimen as in primary conservatively treated patients (Fig. 1). Recent long-term studies have shown that after resection of benign IPMN, a de novo IPMN in the residual pancreas can be observed in up to $17 \%$
[20]. A lifelong observative approach every 1 to 2 years is therefore recommended by some authors.

The prognosis of the resected noninvasive MCN and SPN is excellent and patients are considered cured after resection. Therefore, current guidelines do not recommend follow-up of these patients. De novo recurrences and a multifocal manifestation like in IPMN are not observed in MCN and SPN. In patients with malignant invasive MCN or SPN, follow-up is equivalent to that of ductal pancreatic carcinoma [7, 21].

Funding Open access funding provided by Medical University of Vienna.

Conflict of interest K. Sahora and M. Schindl declare that they have no competing interests.

Open Access This article is distributed under the terms of the Creative Commons Attribution 4.0 International License (http://creativecommons.org/licenses/by/4.0/), which permits unrestricted use, distribution, and reproduction in any medium, provided you give appropriate credit to the original author(s) and the source, provide a link to the Creative Commons license, and indicate if changes were made. 


\section{References}

1. Laffan TA, etal. Prevalence of unsuspected pancreatic cysts on MDCT.AJR Am J Roentgenol. 2008;191(3):802-7.

2. Brugge WR, et al. Cystic neoplasms of the pancreas. NEngl J Med. 2004;351(12):1218-26.

3. Ferrone CR, et al. Current trends in pancreatic cystic neoplasms. Arch Surg. 2009;144(5):448-54.

4. Tanaka M, et al. International consensus guidelines 2012 for the management of IPMN and MCN of the pancreas. Pancreatology. 2012;12(3):183-97.

5. Adsay NV. Cystic neoplasia of the pancreas: Pathology and biology. J Gastrointest Surg. 2008;12(3):401-4.

6. Scheiman JM, Hwang JH, Moayyedi P. American gastroenterological association technical review on the diagnosis and management of asymptomatic neoplastic pancreatic cysts. Gastroenterology. 2015;148(4):824-48.e22.

7. Crippa S, et al. Mucinous cystic neoplasm of the pancreas is not an aggressive entity: Lessons from 163 resected patients. Ann Surg. 2008;247(4):571-9.

8. Fernandez-Del Castillo C. Mucinous cystic neoplasms. J Gastrointest Surg. 2007;12(3):411-3.

9. Fernandez-Del Castillo C, Adsay NV. Intraductal papillary mucinous neoplasms of the pancreas. Gastroenterology. 2010;139(3):708-13.713.e1-2.

10. Sahora K, Fernandez-del Castillo C. Intraductal papillary mucinous neoplasms. Curr Opin Gastroenterol. 2015;31(5):424-9.

11. Sahora K, et al. Branch duct intraductal papillary mucinous neoplasms: Does cyst size change the tip of the scale? A critical analysis of the revised international consensus guidelines in a large single-institutional series. Ann Surg. 2013;258(3):466-75.

12. Sahora K, et al. Not all mixed-type intraductal papillary mucinous neoplasms behave like main-duct lesions: Im- plications of minimal involvement of the main pancreatic duct. Surgery. 2014;156(3):611-21.

13. Mino-Kenudson M, et al. Prognosis of invasive intraductal papillary mucinous neoplasm depends on histological and precursor epithelial subtypes. Gut. 2011;60(12):1712-20.

14. Al-Haddad M, El Hajj II, Eloubeidi MA. Endoscopic ultrasound for the evaluation of cystic lesions of the pancreas. J Pancreas. 2010;11(4):299-309.

15. Park WG, et al. Diagnostic performance of cyst fluid carcinoembryonic antigen and amylase in histologically confirmed pancreatic cysts. Pancreas. 2011;40(1):42-5.

16. Park WG, Chari ST. Management of pancreatic cystic neoplasms: Decision-making with limited information. Pancreatology. 2010;10(2/3):142-3.

17. Tanaka M, et al. Revisions of international consensus Fukuoka guidelines for the management of IPMN of the pancreas. Pancreatology. 2017;17(5):738-53.

18. Sahora K, et al. Effects of comorbidities on outcomes of patients with Intraductal papillary mucinous neoplasms. Clin Gastroenterol Hepatol. 2015;13(10):1816-23.

19. Pergolini I, et al. Long-term risk of pancreatic malignancy in patients with branch duct Intraductal papillary mucinous neoplasm in a referral center. Gastroenterology. 2017;153(5):1284-1294.el.

20. Xourafas D, Tavakkoli A, Clancy TE, Ashley SW. Noninvasive intraductal papillary mucinous neoplasms and mucinous cystic neoplasms: Recurrence rates and postoperative imaging follow-up. Surgery. 2015;157(3):473-83. https:// doi.org/10.1016/j.surg.2014.09.028.

21. Thompson LD, et al. Mucinous cystic neoplasm (mucinous cystadenocarcinoma of low-grade malignant potential) of the pancreas: A clinicopathologic study of 130 cases. Am J Surg Pathol. 1999;23(1):1-16.

Publisher's Note Springer Nature remains neutral with regard to jurisdictional claims in published maps and institutional affiliations. 